\title{
The Management of Reaction.
}

\author{
GORDON A. RYRIE:
}

CASE of lepra reaction may present symptoms so
insignificant from the point of view of the patient's
comfort that little attention is required outside a temporary stoppage of specific treatment. On the other hand the condition may be so severe that prolonged hospitalisation is essential. Every case of reaction is essentially a case of leprotic instability which may be of good or evil import. It is necessary, therefore, by careful enquiry and examination to elicit the casual factors in this disturbance of the symbiosis of tissue and bacillus. A further reason for close observation is that in a large number of cases improper management of the case will frustrate the effects of any specific treatment. Unfortunately it must also be admitted that in hospitals where modern facilities and drugs are not available, it tends to be assumed that nothing can be done for reaction. In such cases reaction tends to be regarded simply as an Act of God and the lepers' enemies-deplorable possibly, but not calling for active intervention. As it is of definite value to pool our experiences of leprosy work apart from specific accounts of drugs and statistics, it may be of interest to outline the general methods of management of a case of reaction as they exist in Sungei Buloh.

As far as possible even mild cases of reaction are treated in hospital, and preliminary stress is laid on careful enquiry and examination to elicit the precipitating factor. Workers of greater experience than myself will agree that it is unfortunately necessary to point out that a full clinical re-examination of the patient is essential and that such an examination cannot be conducted with rubber gloves on. In Sungei Buloh the vast majority of cases are precipitated by one or other of the following causes :-

1. Over dosage, over prolonged treatment or abrupt cessation of treatment with hydnocarpus derivatives.

2. Concurrent disease. In the last year over $60 \%$ of patients who developed reaction had a positive Wasserman and Kahn. Mild helminthic infections appear to be a common cause. Chronic sepsis is one of the most frequent precipitating factors here.

3. Constipation.

4. Most Chinese and Malays, and many Indians attribute reaction to certain articles of diet. Prawns, shell-fish, ducks eggs, hot curries, snakes flesh, the flesh of an old fowl and bamboo shoots are the most frequently indicted. Possibly some of these are merely post-hoc impressions, others may be allergic. 
5. Drug reactions. The effect of potassium iodide is, of course, well known. Patients, however, who supplement their legitimate treatment with Chinese or Ayurvedic medicines or Malay jungleherbs frequently develop a severe type of reaction. It is difficult to elicit a history in these cases.

6. Emotional Stress. Among womèn domestic strife before or during menstruation, the death of relatives, worry over debts or infidelities, are not infrequent precipitants of reaction.

Onset.-Cutaneous reaction begins as a rule here with a few days of mild localised nerve pain followed by the appearance of one or two isolated spots. These spots may $(a)$ die away in abortive cases, $(b)$ coalesce into spreading tuberculoid lesions, $(c)$ become more or less generalised with fever as in the typical reaction case.

An interesting feature at this point and one that is sometimes of diagnostic value, is the absence of urinary calcium in about $20 \%$ of cases. As the condition improves calcium again appears in the urine. This is not due to any calcium deficiency but to an alteration in the mechanism of phosphatic and calcium excretion. The serum calcium in uncomplicated reaction is within normal limits. The test is very simple. To a test-tube of clear acid urine add a few grains of ammonium oxalate. If the normal calcium is present the urine turns cloudy owing to the formation of insoluble calcium oxalate. If the urine remains clear the patient is on the reaction threshold. By this means it is sometimes possible to detect a reaction before the spots appear. It should be remembered, of course, that the test is not specific and only applies to a limited number of cases. Sometimes, however, it is very useful.

A typical reaction here tends to fall into three stages which require different management. There is a first stage with more or less continuous but irregular fever and with pain and tenderness of the rose spots. In the second stage the temperature tends to be subnormal in the morning, rising to $100 \mathrm{deg}$. or $101 \mathrm{deg}$. F. in the evening. The rose spots may now present the appearance of purulent superficial blebs, which are really rich concentrates of mycobacterium lepræ and very useful for demonstration slides. There may be a difficult third stage with little or occasional fever, but marked by bone pains, joint pains, and increasing stiffness. The process may be aborted by lysis or crisis at any point.

During the first stage the patient is confined to bed on "absolute" rest and given a saline purge. The sedimentation test is over fifty-if not, the case is much more likely to be rapidly spreading tuberculoid leprosy. The 
patient is kept on a diet of bread, milk, eggs and fruit. The diet is of great importance, as treatment frequently fails because the patient is eating extraneous matter supplied by friends and relatives. In a series of cases of reaction treated with mercurochrome some time ago, it was found that while a number of dramatic recoveries were obtained in the male wards no single case appeared to be deriving benefit in the female section. It was found that among the females there was a regular custom of friends bringing in little dishes which they considered tasty or " cooling." When this practice was stopped the same percentage of recoveries was obtained as in the male wards. In any case of obstinate reaction therefore a rigid supervision of diet may be found to be of real help.

At this stage the question sometimes arises as to whether the reaction itself or the precipitating factor should be treated first. Sometimes the wealth of material elicited is embarrassing. In a recent case a young Chinese woman developed reaction after a violent quarrel with a neighbour. On examination, she was found to have gross pyorrhœa, a two-finger spleen, an helminthic infection, a retroverted uterus and a positive Wassermann. Except in cases where the probable precipitating factor is easily removed, I think it better to treat the reaction first. Where the reaction goes on for three weeks without responding to specific treatment the precipitating factor is dealt with in spite of the fact that this may cause a temporary exacerbation of the reaction.

A further feature of this first stage is the occasional appearance of a double peak in the temperature chart within twenty-four hours. This is similar to the double rise seen in kala-azar and S.T. malaria.

During the second stage of swinging temperature the patient may complain of feeling cold and may require an extra blanket. During this stage as well as in the first, plenty of Imperial drink seems to help the patient. We have found it advisable to treat the skin with an oily or glycerine emulsion as soon as the tenderness of the spots has subsided sufficiently to allow its application. The purulent blebs and broken sores are best cleaned with eusol or chloramine T. Sores on the back are covered with patches of gauze sealed round the edge with collodion. Calamine or dusting powders are unsuitable agents for reaction sores as they form a crust under which the pus accumulates. An occasional application of antiphlogistine 
is sometimes helpful in those cases where the sores tunnel in the superficial fascia.

In the third stage of post-reaction pain the patient is given full diet usually with the addition of liver soup. The important part of the management at this point is to ensure free and regular exercise of the smaller joints to prevent stiffening. With children, jig-saw puzzles and plasticene are useful for this purpose. Sleeplessness, owing to aching of bones and joints, pains along the leg veins or chronic malaise, may cause difficulty. An evening round is often helpful as the patient can frequently get a good night's sleep after a little suitable " therapeutic talk." We have found Dovers powder useful so long as the patient does not get drug-minded.

Treatment with hydnocarpus derivatives is not resumed for a full month after the reaction has subsided. In cases where the reaction has subsided, but where prolonged treatment for the precipitating factor is still necessary, no further leprosy treatment is given till this has been removed. 